\title{
Optical transmittance of 2D macroporous silicon structures
}

\author{
L.A. Karachevtseva, O.O. Lytvynenko, E.O. Malovichko and O.J. Stronska \\ Institute of Semiconductor Physics, 45 Nauki Prosp., Kyiv-03028, Ukraine \\ Tel.: 265 9815, Fax: 265 8243, E-mail:kartel@mail.kar.net
}

\section{E.V. Busaneva and O.D. Gorchinsky}

T.Shevchenko Kyiv National University, 64 Volodymyrska Str., Kyiv-03022, Ukraine

\begin{abstract}
Optical transmittance by 2D macroporous silicon structures was investigated in direction parallel to pores. Absolute photonic band gap was measured for wavelengths between one and two optical periods of macroporous silicon structure. For wavelengths less than optical period of macropores there are an essential reduction in transmittance of electromagnetic radiation to (3-6) $10^{-3}$ apparently the homogeneous material and the step formation. Transmission spectra of macroporous silicon as well as steps were explained by a model of directed and decay optical modes formed by macroporous silicon as a short wave-guide structure with a large contrast in dielectric constants of the macropore and the silicon matrix.
\end{abstract}

Keywords: macroporous silicon, optical transmittance, photonic band gap, wave-guide.

Paper received 25.07.01; revised manuscript received 18.10.01; accepted for publication 12.12.01.

\section{Introduction}

Optical transmittance of $2 \mathrm{D}$ periodical photonic structures was investigated theoretically and experimentally in [1-10]. Especially, transverse and longitudinal electromagnetic wave components were measured and calculated for 2D macroporous silicon in direction perpendicular to pores [58]. For E-polarized light in (10)-direction an opaque region was observed between 1 and 2 zones and 3-4 zones [5]. But in [1] there was found that E-polarized radiation was not transmitted by the 3 band of a square lattice of dielectric cylinders due to symmetry constraints. Neglecting the gap region, the overall transmittance decreases with increasing wave numbers from $20 \%$ at $50 \mathrm{~cm}^{-1}$ to $2 \%$ at $500 \mathrm{~cm}^{-1}$. This decrease was explained in terms of scattering of rough surfaces. Really, transmitted intensity decreased by factor 3 in the $30 \mathrm{~mm}$ range and it stayed approximately constant inside the gap in the range $40 \mu \mathrm{m}$ when metal cone behind the sample was removed. So, in accordance to [5] macroporous silicon structures are effective media for long waves and strong scatters for short electromagnetic waves.
Optical transmittance of 2D periodic structures was investigated for parallel to rods (out-of-plane) direction theoretically only [9-11]. By the use of the plane wave method Maradudin in [9] have calculated the dispersion curves (photonic band structure) of electromagnetic wave propagation in a structure consisting an infinite array of parallel, long triangular lattice of air cylinders. The propagation vector $k$ of electromagnetic waves in this structure has a nonzero component $k_{z}$ parallel to rods. The structure studied numerically posses a band gap between 3 and 4 zones common to waves of both E- and H-polarization for propagation in plane parallel to the rods. A new absolute band gap opens up below the first band for non-zero $k_{z}$, whose width increases linearly with increasing $k_{z}$. In [11] the transmission of electromagnetic waves propagating in $2 \mathrm{D}$ photonic crystals for out-of-plane incident angle as high as $85^{\circ}$ was studied. There is a full calculated photonic band gap for both E-and H-polarization for dielectric constant ratio higher than 12.25. So the aim of our work is experimental investigation of optical transmittance by 2D macroporous silicon structures in direction parallel to pores. 


\section{L.A. Karachevtseva et al.: Optical transmittance of 2D macroporous...}

\section{Experimental}

The starting material consisted of $n$-type silicon (100) with $2-5 \Omega \cdot \mathrm{cm}$ resistivity. Macropores with diameters $D_{p}=1-15$ micron were formed due to the generation and transfer of nonequilibrium holes to the $n-S i$ electrochemically treated surface as a result of the optical band-to-band electron-hole generation [12-13]. The electrolyte was 5 weight percent hydrofluoric acid. Parameters of samples are presented in Table 1.

Optical transmittance was measured using IR Fourier spectrometer IFS-113, IR spectrophotometer Specord M85 and He-Ne laser. Absorption coefficient $\alpha$ was recalculated taking into account the measured absorbance value $I_{0} / I_{p s}\left(I_{0}\right.$ - incident irradiation, $I_{p s}-$ transmitted irradiation) and the adsorbance value of reference silicon plates $I_{0} / I_{\mathrm{Si}}\left(I_{\mathrm{Si}}\right.$ - irradiation intensity, transmitted through reference silicon plate).

\section{Results}

Accodingly to Maradudin results [9] in two-dimensional periodic structure with period $a$ absolute photonic band gap forms at $\lambda=1.5 \lambda_{a}\left(\lambda_{a}-\right.$ is the optical period of the photonic structure, equaled to $\left.\left(a-D_{p}\right) e^{I / 2}+D_{p}\right)$ by $2 \mathrm{D}$ photonic structure with $\varepsilon=13$ and the cylinder volume $V=$ 0.84 . Soucoulis calculations [11] predicted photonic band gap formation common for $\mathrm{H}$-and E-polarization of electromagneticirradiation at $\lambda \approx 1.2 \lambda_{a}$ at $\varepsilon=12.3$ and $V=0.73$. We measured absorption bands at $\lambda=1.5 \lambda_{a}$ (sample 2 from Table), $\lambda=1.1 \lambda_{a}$ (sample 4 , Fig. 1 ) and for $\lambda \approx \lambda_{\mathrm{a}}$ (sample 6 ). Obtained results correlate qualitatively with calculations $[9,11]$. Absolute band gap below the first band was not measured. So macroporous silicon structures are effective media for long electromagnetic waves, as in [5].

Absorption spectra of macroporous silicon with different pore diameters and concentration have common features at $\lambda \leq \lambda_{a}$ (Fig.2a). There is an essential reduction in transmittance of electromagnetic radiation with a shortening of the wavelength. Recalculated absorption coefficient is equaled $300-500 \mathrm{~cm}^{-1}$, as in Fig.2b. Really, for earlier meas-

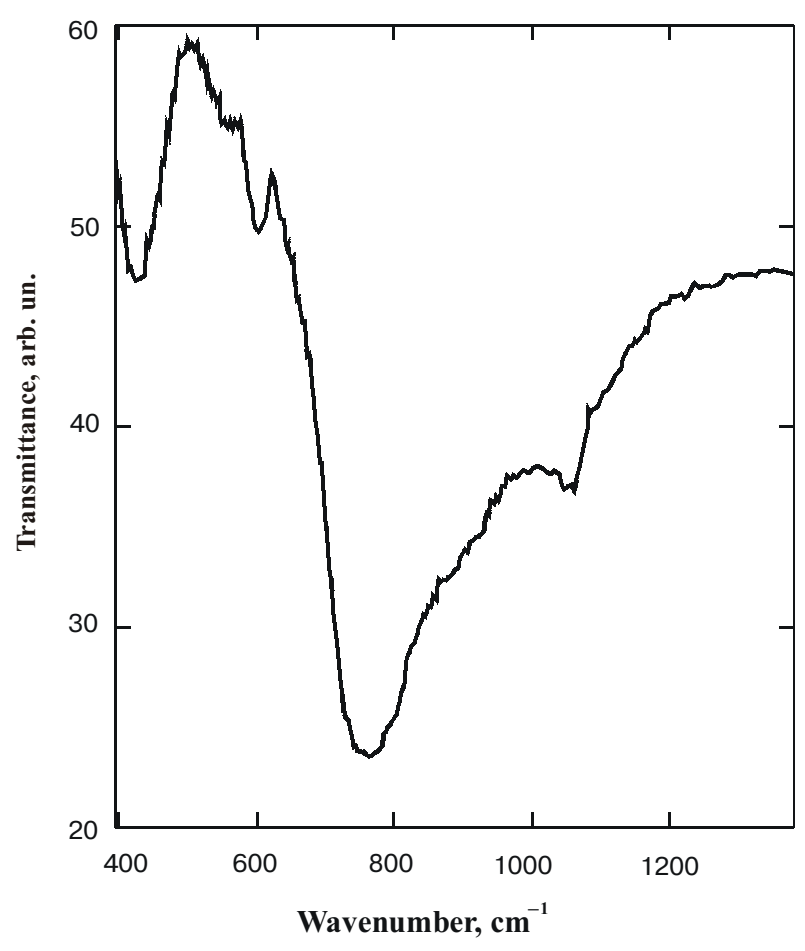

Fig. 1. Transmittance of electromagnetic waves propagating in $2 \mathrm{D}$ macroporous silicon structure parallel to macropores (sample 4).

urements at $\lambda=1.15 \mu \mathrm{m}$ and $\lambda=3.39 \mu \mathrm{m}$ of He-Ne laser optical transmittance of the macroporous structure was 3 $6 \cdot 10^{-3}$ apparently homogeneous material [7]. At short wavelengths $\lambda<D_{p}$ the optical transmittance slightly grows. Transmission spectra contain steps or oscillations, as in Fig. 3. The step frequency in the long-wave part of the spectrum is proportional to the distance between the pores ( $\Delta v^{1} \sim a-D_{p}$, fig. $4 \mathrm{a}$ ), but in the short-wave region it is proportional to the diameter of the pores $\left(\Delta v^{\mathrm{s}} \sim D_{p}\right.$, Fig. $\left.4 \mathrm{~b}\right)$. The frequency intervals of the steps are related via $\Delta v^{\mathrm{s}} / \Delta v^{1} \approx 4$ for the commensurable quantity $a-D_{p}$ and $D_{p}$.

Table. Characteristics of macroporous silicon samples

\begin{tabular}{llllllll}
\hline Sample\# & Type of structure & $h_{p}$, micron & $D_{p}$, micron & $N_{p} \cdot 10^{-5}, \mathrm{~cm}^{-2}$ & $V_{p}$, arb.un. & $a-D_{p}$, micron & $\lambda_{a}$, micron \\
\hline 1 & Arbitrary & 80 & 1 & 75 & 0.06 & 2.8 & 10.8 \\
\hline 2 & Periodical & 70 & 2 & 44 & 0.14 & 2.8 & 11.1 \\
\hline 3 & Arbitrary & 140 & 2.5 & 47 & 0.23 & 2.1 & 10.3 \\
\hline 4 & Periodical & 75 & 3 & 50 & 0.35 & 1.8 & 8.4 \\
\hline 5 & Arbitrary & 150 & 3.5 & 38 & 0.37 & 1.6 & 9.4 \\
\hline 6 & Periodical & 120 & 5 & 12.5 & 0.25 & 3.9 & 18 \\
\hline 8 & Periodical & 80 & 6 & 15.6 & 0.78 & 2.0 & 13.4 \\
\hline 9 & Arbitrary & 170 & 10 & 6.3 & 0.50 & 2.6 & 18 \\
\hline \hline
\end{tabular}




\section{L.A. Karachevtseva et al.: Optical transmittance of 2D macroporous...}

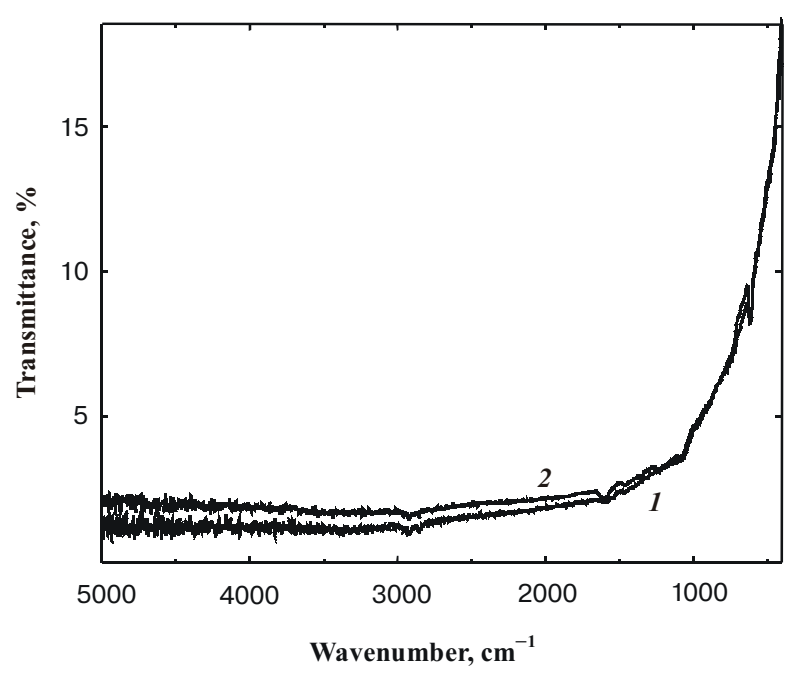

(a)

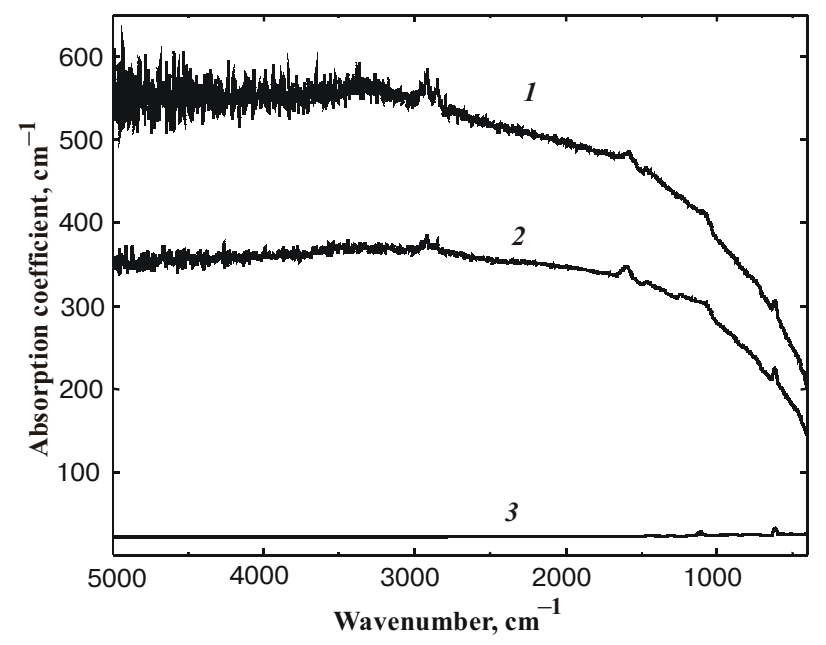

(b)

Fig. 2. (a) Transmittance spectra of macroporous silicon structures: 1 -sample 1, 2 - sample 3. (b) Spectral distribution of the effective absorption coefficient: 1 - sample 1,2-sample 3, 3- silicon plate.

Transmission spectra of macroporous silicon as well as the formation of the steps can be explained by a model of directed and radiated optical modes. Macroporous silicon is a short wave-guide structure with a large contrast in dielectric constants of the pores and the silicon matrix (Fig. 5). The full electromagnetic field of optical waveguides can be represented as the sum of fields of directed modes, transferring energy of radiation over a infinite distance in a spatially stable regime, and fields of radiation in a spatially nonstable regime [14-16]. For short wave-guides, the second component plays a vital role. Thus the field of radiation consists of flowing (refracting and tunnelling) modes and spatial modes of radiation. The basic difference between the directed modes and radiated modes lies in the establishment of the latter, requiring the field to vanish for the distance $z \rightarrow \infty$ [15]. The modes of radiation correspond to frequencies lower than the cut-off frequency. For real values of the longitudinal

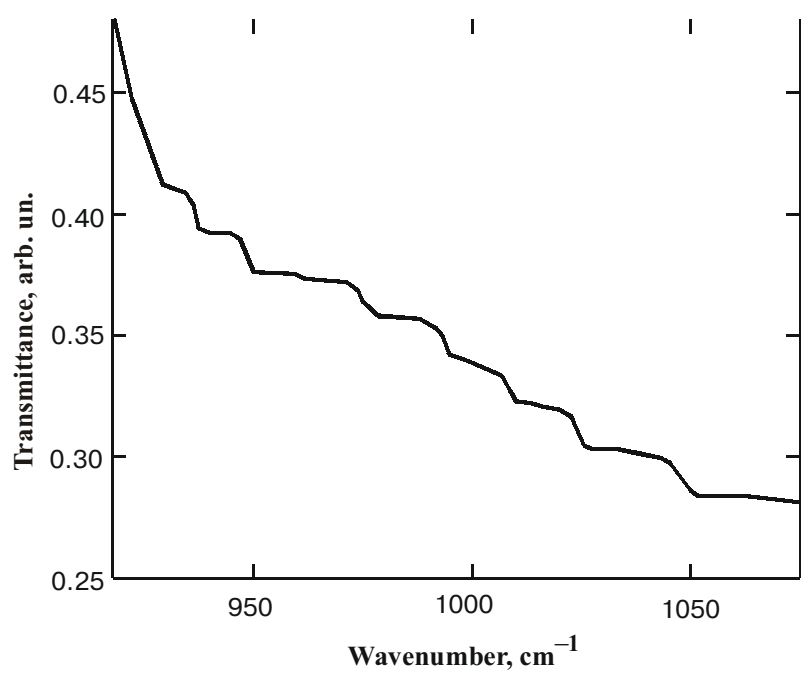

(a)

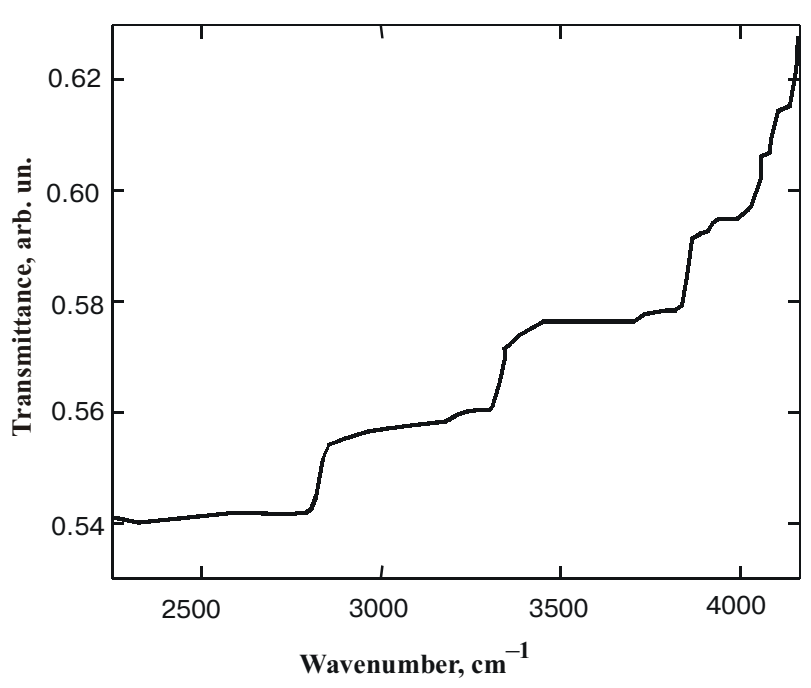

(b)

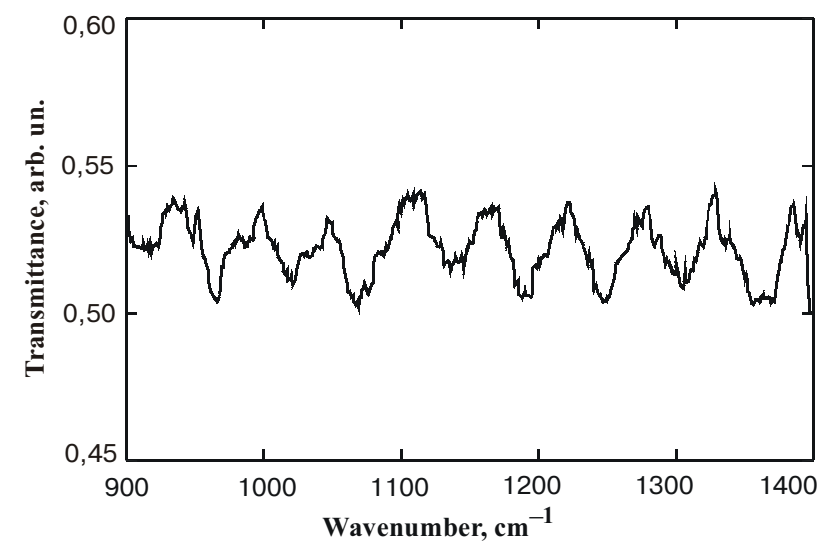

(c)

Fig. 3. (a) Long-wave steps in the transmission spectrum of the sample 5. (b) Short-wave steps in the transmission spectrum of the sample 5. (c) Oscillations in the transmission spectrum of the sample 1. 


\section{L.A. Karachevtseva et al.: Optical transmittance of 2D macroporous...}
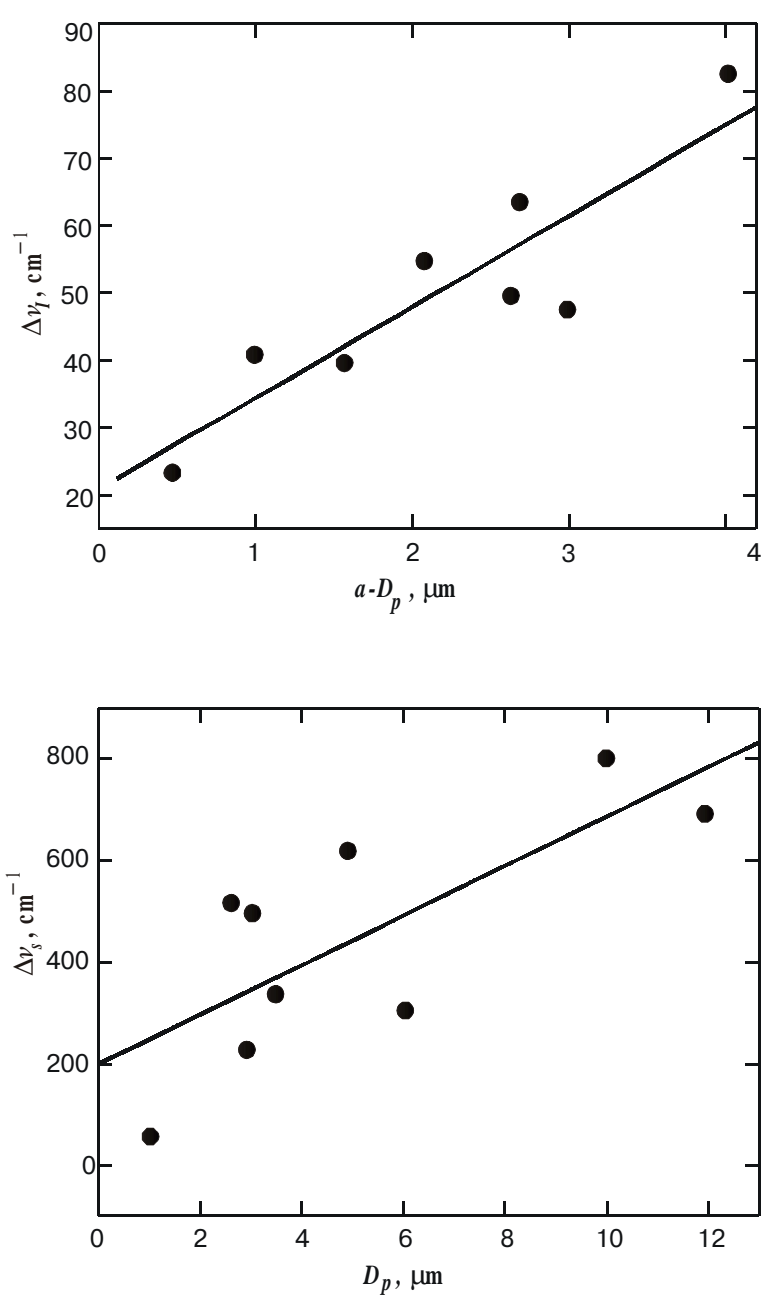

Fig. 4. (a) Dependency of the long-wave step frequency $\Delta v^{1}$ on the distance between pores $a-D_{p}$. (b) Dependency of the shortwave step frequency $\Delta v^{\mathrm{s}}$ on the pore diameter $D_{p}$. (z-axis) component $\beta$ of the wave-vector $k_{2}$ in cladding $\left(0<\beta<k_{2}\right)$ the «propagation» mode of radiation is formed, and the parameter of the mode $Q=\rho\left(\beta^{2}-k_{2}^{2}\right)^{1 / 2}$ in the cladding is imaginary. The radiation mode is defined by a modified cylindrical Bessel function $K_{n}(Q)$ and corresponds to the scattering waves moving away from the waveguide. If the real and imaginary part of $\beta$ satisfy the corresponding conditions $\beta^{r}=0$ and $0<\beta^{i}<\infty$, then the mode is called «decay». For the imaginary value of $b$ the mode parameter $W=\rho\left(k_{1}^{2}-\beta^{2}\right)^{1 / 2}$ is a real quantity for $k_{2}^{2}<$ $<\left|\beta^{2}\right|<k_{1}^{2}$ and corresponds to the directed mode with diminishing amplitude, proportional to $I^{p}(W) e^{-\beta \mathrm{z}}$, where $I^{p}(W)$ - modified Bessel function [16].

In the short-wave spectral region the directed optical modes form on macropores, because the step growth of transmittance takes place for $\lambda \leq D_{p}$ (fig.3b). So macropores present themselves as wave-guides of a metal type (the walls of the macropores are enriched with electrons [17]). For normal incidence of electromagnetic irradiation on macroporous structures there might be sliding radiation incidence on macropore walls. It is known, that for sliding incidence of radiation on conductive surface the absorption coefficient for TM (p) waves is $10^{3}-10^{4}$ times greater of absorption coefficient for TE (s) waves [16]. So TE modes are formed on conductive macropore walls with order $n=1$, because the first directed mode is formed for $\lambda=D_{p}$ [14].

In the middle region $D_{p}<\lambda<\lambda_{a}$ it is possible the directed mode formation for the silicon wave-guides and decay mode formation for the macropores. Such modes are formed in the same medium (the silicon matrix) and differ by the sign of the radius $\rho$ only (Fig. 5), so the formation of shelves is possible. The amplitude of the total wave in the direction $z$ is defined by a cylindrical functions $J_{n}{ }^{S i}(Q) e^{i \beta z}+$ $+I_{n}^{p}(-Q) e^{-\beta z}$.

In the long-wave spectral region $\lambda>\lambda_{a}$ decay apparently Si matrix modes are formed with the mode parameter $Q_{S i}=i k \rho_{S i} n_{S i}\left(\cos \vartheta z^{2}-1\right)^{1 / 2}$.
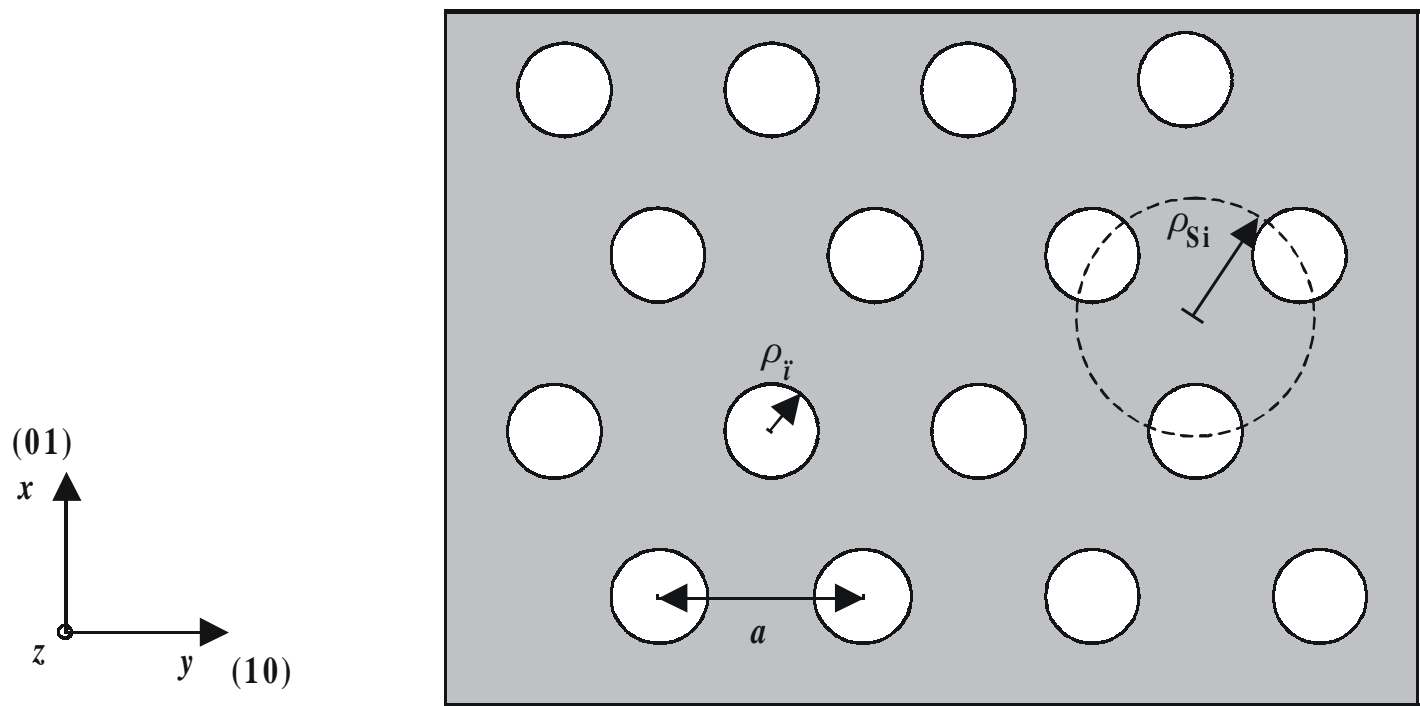

Fig. 5. Cross section of macroporous silicon structure with pore radius $\rho_{p}$ and radius of wave-guide on silicon matrix $\rho_{S i}$. 


\section{L.A. Karachevtseva et al.: Optical transmittance of 2D macroporous...}

The increase in the diameter of macropores up to the value $D_{p}>\left(a-D_{p}\right) n_{S i}$ modifies the transmission spectrum. In this case the region of the decay mode formation corresponds to $\lambda>D_{p}$, and the directed modes are formed for $\lambda<a-D_{p}$. Thus, in contrast to the structures with pore diameters $D_{p} \sim\left(a-D_{p}\right) n_{S i}$ for samples with large macropore diameters the decay modes are formed on pores, and the directed modes are formed on the silicon matrix. For structures with small pore diameters $\left(D_{p} \approx 1 \mu \mathrm{m}\right)$ the transmission spectrum oscillations in the region of wavelengths $\lambda<\lambda_{a}$ are measured only.

\section{Conclusions}

Absolute photonic band gap was measured at wavelengths between one and two optical periods $\left(\lambda_{a}<\lambda<2 \lambda_{a}\right)$ of macroporous silicon structure in accordance to calculations $[9,11]$. The absorption spectra of macroporous silicon with different diameters and concentrations of pores have common features. For wavelengths less than optical period of photonic structure there are an essential reduction in transmittance of electromagnetic radiation to (3-6) $\cdot 10^{-3}$ apparently the homogeneous material and steps formation. For $\lambda<D_{p}$ the optical transmittance slightly grows. In the short-wave spectral region the directed optical modes form on macropores. In the middle region $D_{p}<\lambda<\lambda_{a}$ it is possible the directed mode formation on the silicon waveguide and the decay mode formation on macropores. In the long-wave spectral region $\lambda>\lambda_{a}$ decay apparently Si matrix modes are formed. The increase in the diameter of the macropores up to the value $D_{p}>$ $>\left(a-D_{p}\right) n_{S i}$ modifies the transmission spectrum, decay modes are formed on pores, and the directed modes are formed on the silicon matrix. So transmission spectra of macroporous silicon as well as the steps were explained by a model of directed and decay optical modes formed by macroporous silicon as a short waveguide structure with a large contrast in dielectric constants of the pore and the silicon matrix.

\section{References}

1. R.D. Meade, K.D. Brommer, A.M. Rappe, J.P. Joannopoulos, Existence of photonic band gap in two dimensions // Appl. Phys. Letters, 61(4), pp. 495-497 (1992).
2. P. R. Villeneuve, M. Piche, Photonic band gaps in two-dimensional square and hexagonal lattices // Phys. Rev. B. 46(8), pp. 4969-4972 (1992).

3. W. M. Robertson, G. Arjavalingam, Measurement of photonic band structure in a two-dimensional periodic dielectric array // Phys. Rev. Lett. 68(13), pp.2023-2026 (1992)

4. K.Inoue, M. Wada, K. Sakoda, M. Hayashi, T. Fukushima and A. Yamanaka, Near-infrared photonic band gap of two dimensional triangular air-rod lattices as revealed by transmittance measurement // Phys. Rev. B, 53(3), pp.1010-1013 (1996).

5. U.Gruning and V. Lehmann, Fabrication of 2D infrared photonic crystals in macroporous silicon, article in Photonic Band Gap Materials, Eds. C. M. Soukoulis, pp. 437-444, Kluwer Academic Publishers (1996).

6. A.Birner, U.Gruning, S. Ottow, A. Schneider, F. Muller, V. Lehmann, H. Foll, and U. Gosele, Macroporous Silicon: A twodimensional Photonic Bandgap Material Suitable for near- Infrared Spectral Range // Phys. Stat. Sol. (a) 165(1), pp.111-117 (1998).

7. L.A. Karachevtseva, O.A. Lytvynenko, and E.J. Stronska, Development and optical characteristics of the macroporous silicon structures // Semiconductor Physics, Quantum Electronics \& Optoelectronics 3, pp. 22-25 (2000).

8. F.Muller, A.Birner, U.Gosele, V.Lehmann, S. Ottow, and H.Foll, Structuring of Macroporous Silicon for Application as Photonic Crystals // Journal of Porous Materials, 7, pp.201-204 (2000).

9. Maradudin, A.R. McGurn, Out of plane propagation of electromagnetic waves in a two-dimensional periodic dielectric medium // J. Mod. Opt. 41 (2), pp. 275-284 (1994).

10. T. A Birks, D. M. Atkin, G. Wylangowski, P. Russel, P. Roberts, 2D Photonic Band Gap Structure in Fibre Form, article in Photonic Band Gap Materials, Eds. C. M. Soukoulis, pp. 437444, Kluwer Academic Publishers (1996).

11. M.M. Sigalas, R. Bismas, K.M. Ho, and Soukoulis, Theoretical investigation off-plane propagation of electromagnetik waves in two-dimensonal photonic crystals // Phys. Rev. B,58(11), pp.6791-6794 (1998).

12. V. Lehmann, H. Foll, Formation Mechanism and Properties of Electrochemically Etched Trenches in n-Type Silicon // $J$. Elektrochem. Soc. 137(2), pp. 653-659 (1990).

13. Karachevtseva, O. A. Litvinenko, E. A. Malovichko, Stabilization of Electrochemical Formation of Macropores in $n$-Si // J.Theor.and Experim.Chem. 34(5), pp. 314-318 (1998).

14. M.J.Adams, An Intorduction to Optical Waveguide, John Wiley and Sons, Chichester-N.York, Brisbane, Toronto, 1981, 512p.

15. A.W.Snyder, J.D.Love, Optical Waveguide Theory, London, New York, Charman and Hall, 1983, 656p.

16. S. Solimeno, B. Crosignani, P. DiPorto, Guiding, Diffraction and Confinement of Optical Radiation. Academic Press, New York, 1986, $664 \mathrm{p}$.

17. R.Yu.Holiney, L.A.Matveeva, E.F.Venger, O.A.Litvinenko, and L.A.Karachevtseva, Electroreflectance study of macroporous silicon structures // Appl. Surface Sci. 172(3), pp.214-219 (2001). 